\title{
Efeito do triadimenol sobre o desenvolvimento do feijoeiro (Phaseolus vulgaris L.) e atividade microbiológica do solo
}

\author{
Effect of triadimenol on the development of bean \\ (Phaseolus vulgaris L.) and soil microbial activity
}

Matheus Fonseca de Souza ${ }^{1}$, Ruimário Inácio Coelho $^{2}$, Olavo dos Santos Pereira Junior
Vanderlan de Oliveira Paulucio
, Eldelon de Oliveira Pereira

\section{RESUMO}

O triadimenol é um fungicida sistêmico, pertencente ao grupo dos triazóis, aplicado principalmente via solo no controle de diversas doenças de plantas. Esse trabalho teve como objetivo avaliar o efeito do triadimenol na cultura do feijoeiro e atividade microbiológica do solo submetido a diferentes concentrações de triadimenol. Os estudos foram realizados no laboratório de química e na casa de vegetação, instalados no CCA/Alegre/ES. O delineamento experimental adotado foi o inteiramente casualizado com 6 tratamentos, com 4 repetições utilizando 2 vasos por repetição. Os tratamentos utilizados foram: T1 =0,527 g de baysiston ${ }^{\circledR} .(170 \mathrm{~kg} / \mathrm{ha})$; $\mathrm{T} 2=0,124 \mathrm{~g}$ de baysiston ${ }^{\circledR}(40 \mathrm{~kg} / \mathrm{ha}) \mathrm{T} 3=$ testemunha; $\mathrm{T} 4=0,155 \mathrm{~g}$ de baysiston $^{\circledR}(50 \mathrm{~kg} / \mathrm{ha}) ; \mathrm{T} 5=0,217 \mathrm{~g}$ de baysiston $(70 \mathrm{~kg} /$ ha); $\mathrm{T} 6=0,186 \mathrm{~g}$ de baysiston ${ }^{\circledR}(60 \mathrm{~kg} / \mathrm{ha})$. Para maioria das características avaliadas o tratamento $\mathrm{T} 3$ foi estatisticamente superior aos demais tratamentos. Para a atividade microbiológica do solo, a hidrólise de FDA ocorreu em todos os tratamentos com média $244,926 \mu \mathrm{g}$ FDA hidrolisada hora ${ }^{-1} \mathrm{~g}^{-1}$ de solo, mas não houve diferença significativa entre nenhum dos tratamentos.

Palavras chave: Desenvolvimento, feijão, fungicida, microbiologia, sistêmico, triadimenol.

\section{ABSTRACT}

Triadimenol is a systemic fungicide which belongs to the group of triazoles, used mainly for the control of several diseases of plants. The present work aimed to evaluate the effect of triadimenol in the culture of beans. The experimental design was completely randomized; using 6 treatments with 4 replicates and 2 pots per replicate. The treatments were: $T 1=0.527 \mathrm{~g}$ of baysiston ${ }^{\circledR} .(170 \mathrm{~kg} /$ ha); $T 2=0.124 \mathrm{~g}$ of baysiston ${ }^{\circledR}(40 \mathrm{~kg} / \mathrm{ha}) T 3=$ control; $T 4=0.155 \mathrm{~g}$ of baysiston ${ }^{\circledR}(50 \mathrm{~kg} / \mathrm{ha}) ; T 5=0.217 \mathrm{~g}$ of baysiston $(70 \mathrm{~kg} /$ ha) and T6 $=0.186 \mathrm{~g}$ of baysiston ${ }^{\circledR}(60 \mathrm{~kg} / \mathrm{ha})$. For most of the characteristics evaluated the control T3 was statistically superior to other treatments. For soil microbial activity, the average hydrolysis of FDA in all treatments was $244.926 \mu \mathrm{g}$ of FDA hydrolyzed hour ${ }^{-1} g^{-1}$ of soil. Although the test has been shown to be effective, no significant difference was observed between treatments.

Key words: Development, bean, fungicide, microbial, systemic, triadimenol.

\section{Introdução}

Os benefícios advindos da utilização dos agrotóxicos são incontestáveis, porém, sérios problemas surgem com o emprego indiscriminado destes produtos, que podem atingir o solo pela aplicação direta, pelo tratamento de sementes ou pela pulverização das partes aéreas das plantas (Lopes et al., 2002).

As moléculas de pesticidas podem ser absorvidas pelas plantas e outros organismos do solo, transformadas por meios bióticos e abióticos, retidas pelos

1 Mestrando em Solos e Nutrição de Plantas - Universidade Federal de Viçosa, Departamento de solos, Viçosa-MG, Brasil. E-mail: matheus-ufes@hotmail.com

2 Depto. de Produção Vegetal do Centro de Ciências Agrárias-Universidade Federal do Espirito Santo (CCA-UFES). Alegre-ES, Brasil. E-mail: ruimario@cca.ufes.br

3 Depto. de Zootecnia do Centro de Ciências Agrárias-Universidade Federal do Espirito Santo (CCA-UFES). Alegre-ES, Brasil. E-mail: olavouspjr@uol.com.br

4 Centro de Ciências Agrárias-Universidade Federal do Espirito Santo (CCA-UFES). Alegre-ES, Brasil.

E-mail: vopaulucio@yahoo.com.br

5 Mestrando em Produção Vegetal, Centro de Ciências Agrárias-Universidade Federal do Espirito Santo (CCA-UFES), Depto de Produção Vegetal, Alegre-ES, Brasil. E-mail: eldelon_neo@ hotmail.com

6 Mestrando em Produção Vegetal, Centro de Ciências Agrárias-Universidade Federal do Espirito Santo (CCA-UFES), Depto de Produção Vegetal, Alegre-ES, Brasil. E-mail: mqm_agroline@ hotmail.com 
colóides orgânicos e inorgânicos do solo. Embora esses produtos evitem perdas na produtividade das culturas eles podem ter efeito na atividade microbiológica do solo (Nóbrega et al., 2005).

Os microrganismos ocupam 5\% do espaço poroso do solo (Silva et al., 2004), sendo responsáveis direta e indiretamente por processos bioquímicos diversos, os quais exercem enorme influência na produtividade e sustentabilidade dos ecossistemas terrestres (Siqueira et al., 1994).

Entre os pesticidas usados na agricultura brasileira está o triadimenol largamente utilizado na cultura do cafeeiro, em plantas ornamentais e outras (Clive, 1995). É um fungicida sistêmico, pertencente ao grupo dos triazóis, aplicado principalmente via solo no controle de diversas doenças causadas por fungos. No Brasil é usado no controle da Hemileia vastatrix (Gravena et al., 1993), responsável por grandes perdas da produção.

A molécula do triadimenol é adsorvida principalmente na fração orgânica do solo (Lopes, 1999), sendo altamente persistente no solo, em um período que pode variar entre 169 e 294 dias (Carvalho, 2000).

O feijoeiro Phaseolus vulgaris L. é cultivado nos mais variados tipos de solo, clima e sistemas de produção, tais como o cultivo solteiro ou consorciado com uma ou mais espécies (Carvalho et al., 2007).

De acordo com Paulo et al. (2004); Nascimento et al. (2006) o café em muitos casos é cultivado em consórcio com diversas culturas entre as linhas de plantio, predominantemente na agricultura familiar onde além de proteger o solo é uma fonte de alimentação, sendo a cultura do feijoeiro uma das mais utilizadas. É comum em regiões tradicionais produtoras de café os produtores introduzirem a cultura consórcio após a aplicação do triadimenol sem nenhuma preocupação com os possíveis efeitos sobre esta cultura e também na qualidade do produto colhido.

O objetivo deste trabalho foi avaliar o efeito do triadimenol no desenvolvimento da cultura do feijoeiro e avaliar a atividade microbiológica do solo submetido a diferentes concentrações de triadimenol.

\section{Material e Métodos}

O experimento foi instalado em casa de vegetação do Centro de Ciências Agrárias da Universidade Federal do Espirito Santo, município de Alegre/ES, situado a $20^{\circ} 45^{\prime} 48^{\prime \prime}$ de latitude sul e 41 $31^{\prime} 57^{\prime \prime}$ de longitude oeste e a $210 \mathrm{~m}$ de altitude.
Para montagem do experimento foi utilizado latossolo vermelho-amarelo peneirado (peneira de $2 \mathrm{~mm}$ ), como substrato, acrescido de esterco e areia nas proporções de $3: 2: 1$, respectivamente. A mistura foi acondicionada em vasos com volume de $6,28 \mathrm{dm}^{3}$. Imediatamente após o plantio do feijão foi realizada a aplicação de baysiston ${ }^{\circledR}$.

A definição das diferentes concentrações do produto nas soluções empregadas no experimento teve como base a recomendação do fabricante do produto para a cultura do cafeeiro implantada em diferentes densidades de plantio: com até 1000 plantas/hectare, $40 \mathrm{Kg}$ do produto/hectare; 1001 a 4000 plantas/hectare, $50 \mathrm{Kg}$ do produto/hectare; 4001 a 6000 plantas/hectare, $60 \mathrm{Kg}$ do produto/ hectare e 5000 a 10000 plantas/hectare, $70 \mathrm{Kg}$ do produto/hectare. O cálculo da dosagem levou em consideração o volume de solo contido nos vasos utilizados no experimento, tomando por base que a recomendação em nível de campo considera os primeiros $20 \mathrm{~cm}$ da camada arável do solo.

Para a correta irrigação dos tratamentos, a capacidade de retenção de água pelo substrato (latossolo+esterco+areia) foi determinada, de acordo com metodologia recomendada por Casaroli (2008).

$\mathrm{O}$ delineamento experimental utilizado foi o inteiramente casualizado com 6 tratamentos, repetidos 4 vezes utilizando 2 vasos por repetição, com 20 sementes por vaso.

Os tratamentos utilizados foram: $\mathrm{T} 1=0,527 \mathrm{~g}$ de baysiston ${ }^{\circledR}(170 \mathrm{~kg} / \mathrm{ha}) ; \mathrm{T} 2=0,124 \mathrm{~g}$ de baysiston ${ }^{\circledR}(40 \mathrm{~kg} / \mathrm{ha}) \mathrm{T} 3=$ testemunha; T4 $=0,155 \mathrm{~g}$ de baysiston ${ }^{\circledR}(50 \mathrm{~kg} / \mathrm{ha}) ; \mathrm{T} 5=0,217 \mathrm{~g}$ de baysiston $^{\circledR}(70 \mathrm{~kg} / \mathrm{ha}) ; \mathrm{T} 6=0,186 \mathrm{~g}$ de baysiston ${ }^{\circledR}$ (60 kg/ha).

\section{Desenvolvimento do feijoeiro}

Para o cálculo do índice de velocidade de emergência (IVE) foram realizadas contagens diárias do número de plântulas emergidas, a partir da emergência da primeira plântula. O cálculo do IVE foi feito segundo a metodologia proposta por Maguire (1962): $\mathrm{IVE}=\mathrm{E} 1 / \mathrm{N} 1+\mathrm{E} 2 / \mathrm{N} 2+\ldots+\mathrm{En} / \mathrm{Nn}$, em que: IVE = índice de velocidade de emergência; E1, $\mathrm{E} 2, \ldots \mathrm{En}=$ número de plântulas emergidas no dia, computadas na primeira, segunda, ... última contagem; $\mathrm{N} 1, \mathrm{~N} 2, \ldots \mathrm{Nn}=$ número de dias da semeadura à primeira, segunda,... última contagem.

Após o vigésimo dia do inicio do experimento foram retiradas 3 plantas de cada vaso para a análise 
da massa da matéria fresca e massa da matéria seca. A retirada das plantas teve como critério a uniformidade dentro de cada vaso, utilizando-se sempre plantas com altura média. Apenas duas plantas por vaso foram conduzidas até o fim do ciclo.

A altura das plantas foi determinada medindo-se, com uma régua graduada em centímetros, a distância entre o coleto e o ápice caulinar da planta.

A avaliação da massa da matéria fresca na primeira avaliação se deu imediatamente após a obtenção das plantas utilizando-se de uma balança analítica, com quatro casas decimais. Para a avaliação da massa da matéria seca, as plantas foram mantidas em estufa de circulação forçada de ar a uma temperatura de $70{ }^{\circ} \mathrm{C}$ até peso constante. Em seguida a massa seca foi determinada usando a mesma balança empregada para determinação da massa da matéria fresca.

Após o término do ciclo reprodutivo, as duas plantas restantes foram retiradas do vaso e avaliadas em relação ao comprimento das raízes, número de grãos e massa da matéria fresca dos grãos. Para a determinação do tamanho das raízes, as mesmas foram previamente lavadas para retirada do solo, e, com o auxílio de uma régua graduada $(\mathrm{cm})$ foi feita à medição do comprimento entre o colo e o ápice da raiz primária. Para a avaliação final da massa fresca e massa seca empregou-se a metodologia já descrita anteriormente.

\section{Atividade microbiológica do solo}

Após o término do ciclo reprodutivo do feijoeiro (90 dias), foram retiradas as amostras de solo dos vasos e estimado atividade microbiana total pelo método da hidrólise de diacetato de fluoresceína (FDA), como descrito a seguir.

Foram coletados 5 gramas de solo seco peneirado em peneira de $2 \mathrm{~mm}$ e distribuídos em elernmeyer de $125 \mathrm{ml}$, em 4 repetições. Foram acrescentados $20 \mathrm{ml}$ de tampão fosfato $\left(8,7\right.$ gramas de $\mathrm{K}_{2} \mathrm{HPO}_{4}+1,3$ gramas de $\mathrm{KH}_{2} \mathrm{PO}_{4}$ ), $200 \mu \mathrm{l}$ de diacetato de fluoresceína (15 $\mathrm{mg}$ de diacetato $+7,5 \mathrm{ml}$ de acetona solução a $2 \mathrm{mg} /$ litro) e revestido os elernmeyer com folha de alumínio. A mistura foi incubada em agitador a $150 \mathrm{rpm}$ a $32^{\circ} \mathrm{C}$, por 20 minutos. Após esse período de incubação, a reação foi interrompida pela adição de $20 \mathrm{ml}$ de acetona. A suspensão do solo foi centrifugada a $4000 \mathrm{~g}$ por 10 minutos, tomando-se alíquotas do sobrenadante para análise em espectrofotômetro a $490 \mathrm{~nm}$.
Juntamente com as amostras foi preparada uma curva de calibração, para cada amostra a ser ensaiada nas concentrações $0,100,200,300,400 \mu \mathrm{lde}$ diacetato de fluoresceína, previamente hidrolisada por calor. Para isso foi acrescentado $5 \mathrm{ml}$ de tampão fosfato em tubos de ensaio e adicionado solução de diacetato de fluoresceína nas concentrações $(0$, $50,100,150,200 \mu \mathrm{l})$, permanecendo a solução em banho-maria por 5 minutos. Foram pesados $5 \mathrm{~g}$ do mesmo solo, em duas repetições para cada concentração em elernmeyer e adicionado as diferentes concentrações de diacetato de fluoresceína, acrescido de $15 \mathrm{ml}$ de tampão fosfato. Em seguida a curva padrão recebeu o mesmo tratamento das amostras, como descrito acima.

Os dados obtidos foram submetidos à análise de variância. Para a comparação das médias utilizou-se o teste de Tukey a 5\% de probabilidade, empregandose o programa estatístico SAEG 9.1.

\section{Resultado e Discussão}

\section{Desenvolvimento do feijoeiro}

A emergência das plântulas teve início aos seis dias após o plantio. O índice de velocidade de emergência foi estatisticamente superior no tratamento T3 (testemunha) com 3,125 plantas por dia (Quadro 1). O tratamento T1 maior dosagens de baysiston ${ }^{\circledR}(170 \mathrm{~kg} / \mathrm{ha})$ apresentou o menor índice de velocidade de emergência sendo estatisticamente inferior aos demais tratamentos, esse resultado difere do encontrado por Picinini \& Fernandes (2008) trabalhando com diferentes fungicidas na cultura do trigo, onde não observaram nenhuma diferença no índice de velocidade de emergência. Os demais tratamentos apresentaram valores superiores a T1 e inferiores a T3, diferindo significativamente destes, porém não diferindo entre si quanto ao índice de velocidade de emergência (Quadro 1).

Como pode ser observado no (Quadro 1), para característica altura as plantas do tratamento T3 (testemunha), apresentaram valores estatisticamente superior aos demais tratamentos, sendo observado o menor valor médio para as plantas do tratamento T1, ou seja, na maior concentração empregada de baysiston ${ }^{\circledR}(170 \mathrm{~kg} / \mathrm{ha})$ sendo estatisticamente inferior aos demais tratamentos, que não se diferenciaram entre si resultado semelhante ao encontrado por Junior et al. (2008) trabalhando com o triadimenol na cultura do trigo. 
Quadro 1. Valores médios para o IVE em dias, altura (ALT) em cm, massa da matéria fresca da parte aérea (MFA), massa da matéria seca da parte aérea (MSA) em gramas. Em avaliações ocorridas aos 20 dias após a germinação das sementes. CCA-UFES, Alegre-ES, 2009.

\begin{tabular}{lcrll}
\hline TRAT. & IVE em dias & ALT. & \multicolumn{1}{c}{ MFA } & \multicolumn{1}{c}{ MAS } \\
\hline 1 & $1,370 \mathrm{c}$ & $8,250 \mathrm{c}$ & $2,845 \mathrm{c}$ & $0,350 \mathrm{~b}$ \\
2 & $2,116 \mathrm{~b}$ & $17,250 \mathrm{~b}$ & $5,152 \mathrm{bc}$ & $0,633 \mathrm{ab}$ \\
3 (Testemunha) & $3,125 \mathrm{a}$ & $27,000 \mathrm{a}$ & $8,347 \mathrm{a}$ & $0,885 \mathrm{a}$ \\
4 & $2,095 \mathrm{~b}$ & $17,250 \mathrm{~b}$ & $6,390 \mathrm{ab}$ & $0,698 \mathrm{a}$ \\
5 & $1,823 \mathrm{~b}$ & $17,000 \mathrm{~b}$ & $6,145 \mathrm{ab}$ & $0,677 \mathrm{a}$ \\
6 & $2,052 \mathrm{~b}$ & $18,000 \mathrm{~b}$ & $6,427 \mathrm{ab}$ & $0,706 \mathrm{a}$ \\
Média & 2,101 & 17,458 & 5,884 & 0,658 \\
\hline
\end{tabular}

Médias com mesma letra na coluna não diferem entre si pelo teste de Tukey a 5\% Probabilidade.

Com relação massa da matéria fresca da parte aérea os melhores resultados foram para os tratamentos T3, T4, T5 e T6 não diferindo estatisticamente entre si, sendo que apenas o T3 diferi estatisticamente dos tratamentos T1 e T2 (Quadro 1). Comportamento semelhante foi observado para a massa da matéria seca da parte aérea, porém com o tratamento T3 diferindo apenas de T1 (Quadro 1).

$\mathrm{Na}$ avaliação final como pode ser observado na (Quadro 2) o tratamento T3 (testemunha) apresentou altura superior aos demais tratamentos, os menores valores foram observados para o tratamento T1. Quanto a característica massa da matéria fresca aérea e massa da matéria seca aérea os menores valores foram observados para o tratamento $\mathrm{T} 1$ que não diferiu estatisticamente de T2, mas diferiu dos demais tratamentos (Quadro 2). Esse resultado difere do encontrado por Goulart (2008) trabalhando com diferentes fungicidas, onde não observou nenhuma diferença no peso das plantas.

Para as características número de grãos normais, número de grãos com defeito, massa da matéria fresca da raiz, massa da matéria seca da raiz, massa da matéria fresca dos grãos e massa da matéria seca dos grãos, não houve diferença significativa entre nenhum dos tratamentos (Quadro 3), esse resultado diferente do encontrado por Luz \& Pereira (1998) trabalhando com diferentes fungicidas na cultura do milho, onde observaram um aumento de produção com aplicação do fungicida thiran.

\section{Atividade microbiológica do solo}

A hidrólise de FDA ocorreu em todos os tratamentos. A atividade microbiológica foi notadamente
Quadro 2. Valores médios de altura (ALT) em cm, massa da matéria fresca da parte aérea (MFA), massa da matéria seca da parte aérea (MSA) em gramas, número de grãos normais (NGN), número de grãos com defeito (NGD). CCA-UFES, Alegre-ES, 2009.

\begin{tabular}{lrlllr}
\hline TRAT. & ALT. & MFA & MSA & NGN & NGD \\
\hline 1 & $8,250 \mathrm{c}$ & $2,845 \mathrm{c}$ & $0,350 \mathrm{~b}$ & $23,250 \mathrm{a}$ & $8,500 \mathrm{a}$ \\
2 & $17,250 \mathrm{~b}$ & $5,152 \mathrm{bc}$ & $0,633 \mathrm{ab}$ & $34,000 \mathrm{a}$ & $13,500 \mathrm{a}$ \\
3 (Testemunha) & $27,000 \mathrm{a}$ & $8,347 \mathrm{a}$ & $0,885 \mathrm{a}$ & $22,000 \mathrm{a}$ & $11,250 \mathrm{a}$ \\
4 & $17,250 \mathrm{~b}$ & $6,390 \mathrm{ab}$ & $0,698 \mathrm{a}$ & $23,250 \mathrm{a}$ & $8,000 \mathrm{a}$ \\
5 & $17,000 \mathrm{~b}$ & $6,145 \mathrm{ab}$ & $0,677 \mathrm{a}$ & $22,750 \mathrm{a}$ & $11,000 \mathrm{a}$ \\
6 & $18,000 \mathrm{~b}$ & $6,427 \mathrm{ab}$ & $0,706 \mathrm{a}$ & $27,250 \mathrm{a}$ & $12,750 \mathrm{a}$ \\
Média & 17,458 & 5,884 & 0,658 & 25,416 & 10,833 \\
\hline
\end{tabular}

Médias com mesma letra na coluna não diferem entre si pelo teste de Tukey a 5\% Probabilidade.

Quadro 3. Valores médios de massa da matéria fresca da raiz (MFR), massa da matéria seca da raiz (MSR), massa da matéria fresca dos grãos (MFG), massa da matéria seca dos grãos (MSG) todos em gramas. CCA-UFES, Alegre-ES, 2009.

\begin{tabular}{lllll}
\hline TRAT. & MFR & MSR & MFG & MSG \\
\hline 1 & $5,810 \mathrm{a}$ & $0,697 \mathrm{a}$ & $2,740 \mathrm{a}$ & $0,108 \mathrm{a}$ \\
2 & $8,968 \mathrm{a}$ & $1,145 \mathrm{a}$ & $4,104 \mathrm{a}$ & $0,211 \mathrm{a}$ \\
3 (Testemunha) & $7,953 \mathrm{a}$ & $1,380 \mathrm{a}$ & $4,162 \mathrm{a}$ & $0,100 \mathrm{a}$ \\
4 & $7,270 \mathrm{a}$ & $1,073 \mathrm{a}$ & $3,969 \mathrm{a}$ & $0,624 \mathrm{a}$ \\
5 & $6,445 \mathrm{a}$ & $0,626 \mathrm{a}$ & $2,749 \mathrm{a}$ & $0,153 \mathrm{a}$ \\
6 & $8,088 \mathrm{a}$ & $0,901 \mathrm{a}$ & $3,394 \mathrm{a}$ & $0,270 \mathrm{a}$ \\
Média & 7,422 & 0,970 & 3,520 & 0,151 \\
\hline
\end{tabular}

Médias com mesma letra na coluna não diferem entre si pelo teste de Tukey a 5\% Probabilidade.

alta em todos os solos com média de $244,926 \mu \mathrm{g}$ FDA hidrolisada $\mathrm{g}^{-1}$ de solo hora ${ }^{-1}$ (Quadro 4).

A atividade microbiológica atingiu seu maior valor em nossa testemunha $(\mathrm{T} 3=0$ gramas de baysiston ${ }^{\circledR}$ por vaso) com 270,522 $\mu \mathrm{g} \mu \mathrm{g}$ FDA hidrolisada $\mathrm{g}^{-1}$ de solo hora ${ }^{-1}$, resultado $16,96 \%$ superior a maior concentração de baysiston ${ }^{\circledR}$ ( $\mathrm{T} 1=0,527$ gramas baysiston ${ }^{\circledR}$ por vaso) com 231,285 $\mu \mathrm{g}$ FDA hidrolisada $\mathrm{g}^{-1}$ de solo hora ${ }^{-1}$, apesar de estatisticamente serem iguais (Quadro 4). Resultado semelhante foi observado por Oliveira et al. (2007), onde não houve diferença significativa na atividade microbiana entre solos cobertos com caatinga e com palma forrageira.

Todos os demais tratamentos apresentaram valores intermediários entre T1 e T3, mas nenhum dos tratamentos se diferenciou estatisticamente (Quadro 4). 
Quadro 4. Valores médios de $\mu \mathrm{g}$ FDA hidrolisada $\mathrm{g}^{-1}$ de solo hora ${ }^{-1}$. CCA-UFES, Alegre-ES, 2009

\begin{tabular}{lc}
\hline TRAT. & $\mu$ g FDA hidrolisada $\mathrm{g}^{-1}$ de solo hora $^{-1}$ \\
\hline 1 & $231,285 \mathrm{a}$ \\
2 & $243,419 \mathrm{a}$ \\
3 (Testemunha) & $270,522 \mathrm{a}$ \\
4 & $238,528 \mathrm{a}$ \\
5 & $231,717 \mathrm{a}$ \\
6 & $254,084 \mathrm{a}$ \\
Média & $244,926 \mathrm{a}$
\end{tabular}

Médias com mesma letra na coluna não diferem entre si pelo teste de Tukey a $5 \%$ probabilidade.

De acordo com o desenvolvimento da técnica de FDA, foi observado para todos os testes realizados, a presença da hidrólise de diacetato de fluoresceína, o que qualifica esse método como promissor e estável, para análise da atividade microbiológica do solo, como tem sido observado por outros autores (Silva et al., 2004). Alguns autores como Alencar
\& Costa (2000) vem utilizando esse método para avaliar o impacto dos produtos químicos na atividade microbiológica do solo, com registro de redução da atividade microbiológica nos solos cultivados com aplicação de agrotóxicos na irrigação. Outros autores como Godoi (2001), Paulucio (2007) vem utilizando esse método como um indicador da atividade microbiana em solos de áreas degradadas.

\section{Conclusão}

A aplicação de baysiston ${ }^{\circledR}$ reduziu o índice de velocidade de emergência;

A aplicação baysiston ${ }^{\circledR}$ reduziu a altura das plantas;

A produção de grãos não foi influenciada com a aplicação de baysiston ${ }^{\circledR}$;

Para as condições desta pesquisa a aplicação do baysiston ${ }^{\circledR}$ não interfere estatisticamente na atividade microbiológica do solo.

\section{Referências Citadas}

Alencar, F.C.N.; Costa, J.L.S.

2000 Impacto da fungigação na biomassa e atividade microbiológica dos solos. In: Congresso Brasileiro de Fitopatologia, 33, 2000, Belém. Fitopatologia Brasileira, Brasília, 25: 359.

Carvalho, A.J.

2007 Sistemas de produção de feijão intercalado com cafeeiro adensado recém-plantado. Ciência e Agrotecnologia. Lavras, 31 (1): 133-139.

Carvalho, R.F.

2000 Sorção e degradação do fungicida triadimenol em solos representativos do município de Lavras-MG. Dissertação (Mestrado) Universidade Federal de Lavras, Lavras. 54 p.

Casaroli, D; Lier, Q.J.V.

2008 Critérios para determinação da capacidade de vaso. Revista Brasileira de Ciência do Solo. Viçosa, 32 (1): 59-66.

Clive, T.

1995 A world compendium the pesticide manual: incorporating the agrochemicals handbook. $10^{\text {th }}$ ed. Surrey, UK: Crop Protections Publications, p. 1341.

Godoi, L.C.L.

2001 Propriedades microbiológicas de solos em áreas degradadas e recuperadas na região dos cerrados goianos. 87 p. Dissertação (Mestrado em Agronomia) Universidade Federal de Goiás.

Goulart, A.C.P.

2008 Efeito do tratamento de sementes de algodoeiro com fungicidas no controle do tombamento de plântulas causado por Rhizoctonia solani, sob condições de casa de vegetação. Tropical. plant pathology. Brasilia, 33 (5): 394-398.

Gravena, S.; Harada, S.S.; Benetoli, I.; Yamamoto, P.T.

1993 Comparação de estratégias de manejo de pragas e doenças do cafeeiro (Coffea arabica L.), var. Mundo
Novo, na região de Marília, SP. Científica, 21 (1): 139147.

Júnior, G.D; Vechiato, M.H; Menten, J.O.M

2008 Efeito de fungicidas no controle de Fusarium graminearum, germinação, emergência e altura de plântulas em sementes de trigo. Summa phytopathol. Botucatu, 34 (1): 280-283.

Lopes, N.P. et al.

2002 Influência da matéria orgânica na adsorção do fungicida triadimenol pelo solo. Química Nova. Viçosa, 25 (4): 544-547.

Lopes, N.P.

1999 Adsorção do triadimenol por frações de solo associadas a um composto de lixo urbano. 100 p. Dissertação (Mestrado) Universidade Federal de Viçosa, Viçosa.

Luz, W.C.; Pereira, L.R.

1998 Tratamento de sementes com fungicidas relacionado com o controle de patógenos e rendimento de milho. Ciência Rural. Santa Maria, 28 (4): 537-541.

Maguire, J.D

1962 Speed of germination aid in selection and evaluation for seedlings emergence and vigor. Crop Sci., 2 (1): 176-177.

Nascimento, E.A. et al.

2006 Alterações morfofisiológicas em folhas de cafeeiro (Coffea arabica L.) consorciado com seringueira (Hevea brasiliensis Muell. Arg.). Ciencia Rural. Viçosa, 36 (3): 852-857.

Nóbrega, J.C.A.

2005 Retenção do fungicida triadimenol em latossolos em razão da calagem e da fosfatagem. Pesquisa Agropecuária Brasileira. Brasília, 40 (5): 503-511.

Oliveira, V.C. et al.

2007 Atividade microbiana enzimática (FDA) como bioindicadora da qualidade de solos para o monitoramento ambiental em agroecossistemas do semi-árido. Revista Brasileira de Agroecologia. Sergipe, 2 (1): 894-897. 
Paulo, E.M et al.

2004 Comportamento do cafeeiro Apoatã em consórcio com culturas anuais. Bragantia. Campinas, 63 (2): 275-281.

Paulucio, V.O.

Qualidade Química e Biológica de Áreas degradadas pela Extração de Argila, Revegetada com Eucalipto e Leguminosas Inoculadas com Micorrizas. Universidade Estadual do Norte Fluminense, Campos dos GoytacazesRJ, 106 p. (Tese de Doutorado).
Picinini, E.C; Fernandes, J.M.C.

2003 Efeito do tratamento de sementes com fungicida sobre o controle de doenças na parte aérea do trigo. Fitopatologia. Brasileira. Brasilia, 28 (5): 515-520.

Silva, M. et al.

2004 Hidrólise de diacetato de fluoresceína como bioindicador da atividade microbiológica de um solo submetido ao reflorestamento. Ciência Rural. Santa Maria, 34 (5): 1493-1496.

Siqueira, J.O. et al.

1994 Microrganismos e processos biológicos do solo: perspectiva ambiental. Brasília: Emrapa-SPI, 7-81. 